\title{
MULTIGRID METHODS FOR THE BIHARMONIC EQUATION USING SOME NONCONFORMING PLATE ELEMENTS
}

\author{
LIMING MA ${ }^{1}$ and QIANSHUN CHANG ${ }^{2}$
}

(Received 9 October, 1995; revised 8 January, 2001)

\begin{abstract}
In this paper, multigrid methods for solving the biharmonic equation using some nonconforming plate elements are considered. An average algorithm is applied to define the transfer operator. A general analysis of convergence is given.
\end{abstract}

\section{Introduction}

The biharmonic equation is a typical elliptic equation of order four and possesses important theoretical value. The biharmonic equation is a basic mathematical model in structure mechanics and is used in the blending problem, image restoration, domain transforms, and so forth. In the blending problem, given two frame surfaces (or bodies) already located, a smoothly transferring surface is sought to connect the two frame surfaces along a certain boundary. Usually, the "smoothness" means that the blending surface and its tangent plane are continuous until the joint boundary. Thus, the displacement vector of the blending surface satisfies the biharmonic equation. In general, the nonconforming finite element method is applied in solving the biharmonic problem, since the degrees of freedom in each element, and hence the computational complexity, can be reduced.

Multigrid methods are very efficient iterative solvers for systems of algebraic equations arising from finite element and finite difference discretizations of elliptic boundary value problems. In recent years, multigrid methods of nonconforming plate elements have been studied (see, for example, [4, 9]). But, because the finite element spaces associated with nonconforming elements are not nested, that is, $V_{k-1} \not \subset$ $V_{k}$, it is difficult to define the transfer operator in the multigrid method of some

\footnotetext{
'Graduate School, Academia Sinica, Beijing 100039, China.

${ }^{2}$ Academy of Mathematics and Systems Sciences, The Chinese Academy of Sciences, Beijing 100080, China; e-mail: qschang@public.fhnet.cn.net.

(C) Australian Mathematical Society 2001, Serial-fee code 0334-2700/01
} 
nonconforming plate elements, such as the Adini rectangular element, the Morley element, the complete cubic nonconforming element and the Zienkiewicz triangular element. S. C. Brenner (see [4]) has used the idea of taking averages in defining the transfer operator $I_{k-1}^{k}$ for the Morley element. In this paper, we extend this idea to some of the nonconforming finite elements mentioned above and give a simpler convergence proof than Brenner's.

We know that the complete cubic nonconforming plate element is of the same convergence order as the Morley nonconforming element and the Adini element. However, the cubic element has two degrees of freedom fewer than that of the Adini element. Moreover, we can get the convergence order to be $O\left(h^{2}\right)$ when the compensation method is used. So we apply the complete cubic nonconforming plate element to develop an optimal-order multigrid method for the biharmonic equation in this paper.

The paper is organized as follows. We begin with a discussion of the complete cubic nonconforming plate element. In Section 3, the intergrid transfer operator is defined and its two properties are proved. The multigrid algorithm is given in Section 4. In the last section, the error order of the multigrid method is obtained.

\section{The nonconforming complete cubic plate element}

We consider the plate blending problem with clamped boundary condition

$$
\begin{cases}\triangle^{2} u=f & \text { in } \Omega, \\ u=\partial u / \partial n=0 & \text { on } \partial \Omega,\end{cases}
$$

where $u$ denotes displacement, $f \in H^{-l}(\Omega), l=0$ or 1 and is the loading force, $\Omega$ is a rectangular domain and $n$ is the outward normal direction of boundary $\partial \Omega$.

The boundary value problem (2.1) has a unique solution $u \in H^{4-I}(\Omega) \cap H_{0}^{2}(\Omega)$, which satisfies the following elliptic regularity (see [1]):

$$
\|u\|_{H^{4-1}(\Omega)} \leq C(\Omega)\|f\|_{H^{-1}(\Omega)} .
$$

The problem (2.1) can be associated with the following variational problem: Find $u \in H_{0}^{2}(\Omega)$, such that

$$
a(u, v)=(f, v), \quad \forall v \in H_{0}^{2}(\Omega),
$$

where

$$
a(u, v)=\int_{\Omega}\left(\Delta u \Delta v+(1-\sigma)\left(2 u_{x y} v_{x y}-u_{x x} v_{y y}-u_{y y} v_{x x}\right)\right) d x, \quad(f, v)=\int_{\Omega} f v d x,
$$

and $0<\sigma<1 / 2$ is the Possion ratio. 
The problem (2.3) is discretized by the complete cubic nonconforming plate elements. Let $\left\{\tau_{k}\right\}, k \geq 1$, be a family of rectangular elements of $\Omega$, where $\tau_{k+1}$ is obtained by connecting the midpoints of the edges of the rectangular elements in $\tau_{k}$. Then we have $h_{k}=\max \left\{\operatorname{diam} T, T \in \tau_{k}\right\}=2 h_{k+1}$.

Let $V_{k}$ be the complete cubic nonconforming plate element space associated with $\tau_{k}$. Then $v \in V_{k}$ possesses the following properties:

(i) $\left.v\right|_{T}$ is a cubic polynomial for all $T \in \tau_{k}$;

(ii) the degrees of freedom are the values of the functions at the vertices of the rectangular $T \in \tau_{k}$ and the normal derivatives at the midpoints of the edges and the mean values of two derivatives of the third order;

(iii) $v$ and $\partial v / \partial n$ vanish at the vertices and the midpoints on $\partial \Omega$, respectively.

Note that $V_{k-1} \not \subset V_{k}$ (that is, $V_{k}$ is nonnested) and $V_{k} \not \subset H_{0}^{2}$ (that is, $V_{k}$ is nonconforming).

The discrete problem of (2.3) is: Given $f \in L^{2}(\Omega)$, find $u_{k} \in V_{k}$, satisfying

$$
a_{k}\left(u_{k}, v\right)=(f, v), \quad \forall v \in V_{k},
$$

where

$$
a_{k}(u, v)=\sum_{T \in \tau_{k}} \int_{T}\left(\Delta u \Delta v+(1-\sigma)\left(2 u_{x y} v_{x y}-u_{x x} v_{y y}-u_{y y} v_{x x}\right)\right) d x
$$

and

$$
(f, v)=\sum_{T \in \tau_{k}} \int_{T} f v d x .
$$

Define the mesh-dependent energy norm as

$$
\|v\|_{k}:=\sqrt{a_{k}(v, v)}, \quad \forall v \in V_{k} .
$$

From [3], we have

$$
\left\|u-u_{k}\right\| \leq C\left(h_{k}|u|_{H^{3}}+h_{k}^{2}|f|_{4}\right) .
$$

Thus, we modify the problem (2.4) to take the following form: Find $u_{k}^{*} \in V_{k}$, such that

$$
a_{k}\left(u_{k}^{*}, v\right)=\left(f, v^{\prime}\right), \quad \forall v \in V_{k},
$$

where $v^{\prime}$ is a linear interpolation of $v$.

It is easy to prove the inequality

$$
\left\|u-u_{k}^{*}\right\|_{k} \leq C h_{k}|u|_{H^{3}}
$$

Note that we don't need to make the above modification for the Zienkiewicz element and the Adini element, since $V_{k} \subset C^{0}(\bar{\Omega})$. In this paper, $C$ denotes a generic positive constant, which may have different values in different occurrences. 


\section{The intergrid transfer operator and its properties}

The intergrid operator $I_{k-1}^{k}: V_{k-1}+H^{3}(\Omega) \cap H_{0}^{2}(\Omega) \mapsto V_{k}$ is defined as follows. For $v \in V_{k-1}+H^{3}(\Omega) \cap H_{0}^{2}(\Omega)$,

(i) Let $\mathrm{A}$ be a vertex of element $T \in \tau_{k}$ inside $\Omega$. If $\mathrm{A}$ is also a vertex of $\tau_{k-1}$, then

$$
\left(I_{k-1}^{k} v\right)(A):=v(A) .
$$

If $\mathrm{A}$ is the midpoint of the common edges of two rectangular elements $T_{1}$ and $T_{2}$ in $\tau_{k-1}$, then

$$
\left(I_{k-1}^{k} v\right)(A):=\frac{1}{2}\left[\left.v\right|_{T_{1}}(A)+\left.v\right|_{T_{2}}(A)\right] .
$$

If $\mathrm{A}$ is in the interior of a rectangular in $\tau_{k-1}$, then

$$
\left(I_{k-1}^{k} v\right)(A):=\left.v\right|_{\tau_{k-1}}(A) \text {. }
$$

(ii) If the midpoint $B$ of an edge in an element of $\tau_{k}$ is located in the interior of a rectangular in $\tau_{k-1}$, then

$$
\frac{\partial\left(I_{k-1}^{k} v(B)\right)}{\partial n}:=\left.\frac{\partial v}{\partial n}\right|_{\tau_{k-1}}(B) .
$$

If $B$ is on the common edges of two elements $T_{1}$ and $T_{2}$ in $\tau_{k-1}$ then

$$
\frac{\partial\left(I_{k-1}^{k} v(B)\right)}{\partial n}:=\frac{1}{2}\left[\left.\frac{\partial v}{\partial n}\right|_{T_{1}}(B)+\left.\frac{\partial v}{\partial n}\right|_{T_{2}}(B)\right] .
$$

(iii) $I_{k-1}^{k} v:=0$ at the vertices of $\tau_{k}$ along $\partial \Omega$ and $\partial\left(I_{k-1}^{k} v\right) / \partial n=0$ at the midpoints of the edges of $T \in \tau_{k}$ along $\partial \Omega$.

(iv) Let

$$
\int_{T} \frac{\partial^{3}\left(I_{k-1}^{k} v\right)}{\partial x_{1}^{3}} d x_{1} d x_{2}=\int_{T} \frac{\partial^{3} v}{\partial x_{1}^{3}} d x_{1} d x_{2}
$$

and

$$
\int_{T} \frac{\partial^{3}\left(l_{k-1}^{k} v\right)}{\partial x_{2}^{3}} d x_{1} d x_{2}=\int_{T} \frac{\partial^{3} v}{\partial x_{2}^{3}} d x_{1} d x_{2}, \quad T \in \tau_{k} .
$$

Thus, we have the following two lemmas.

LEMMA 3.1 (Stability Property). For any $v \in V_{k-1}+H^{3}(\Omega) \cap H_{0}^{2}(\Omega)$, there exists a constant $C$ independent of $h_{k}$, such that

$$
\left\|I_{k-1}^{k} v\right\|_{k} \leq C\|v\|_{k-1} .
$$


PROOF.

$$
I_{k-1}^{k} v=\left(I_{k-1}^{k} v-\pi_{k} v\right)+\left(\pi_{k} v-v\right)+v
$$

where $\pi_{k}$ is an interpolation operator from $H^{3}(\Omega) \cap H_{0}^{2}(\Omega)$ to $V_{k}$. Thus, one gets

$$
\left\|I_{k-1}^{k} v\right\| \leq\left\|I_{k-1}^{k} v-\pi_{k} v\right\|_{k}+\left\|\pi_{k} v-v\right\|_{k}+\|v\|_{k-1} .
$$

Using the interpolation theorem of Sobolev space and the inverse inequality, we obtain

$$
\left\|v-\pi_{k} v\right\|_{k} \leq C\|v\|_{k-1} .
$$

So, the key step is to estimate $\left\|I_{k-1}^{k} v-\pi_{k} v\right\|_{k}$.

From the definition of $\pi_{k} v$ and $I_{k-1}^{k} v$, we see

$$
\begin{aligned}
\left.\left(\pi_{k} v-I_{k-1}^{k} v\right)\right|_{T}= & \frac{N_{2}}{2}\left(\left.v\left(A_{2}\right)\right|_{T_{1}}-\left.v\left(A_{2}\right)\right|_{T_{2}}\right)+\frac{N_{4}}{2}\left(\left.v\left(A_{4}\right)\right|_{T_{1}}-\left.v\left(A_{4}\right)\right|_{T_{2}}\right) \\
& +\frac{M_{2}}{2}\left(\left.\frac{\partial v\left(B_{2}\right)}{\partial n}\right|_{T_{1}}-\left.\frac{\partial v\left(B_{2}\right)}{\partial n}\right|_{T_{3}}\right)+\frac{M_{4}}{2}\left(\left.\frac{\partial v\left(B_{4}\right)}{\partial n}\right|_{T_{1}}-\left.\frac{\partial v\left(B_{4}\right)}{\partial n}\right|_{T_{3}}\right),
\end{aligned}
$$

where $T \in \tau_{k}, T_{i}(i=1,2,3)$ are three neighboring elements in $\tau_{k-1}, M_{i}$ and $N_{i}$ $(i=2,4)$ are the shape functions, $A_{i}(i=1,2,3,4)$ are the vertices of $T$ and $B_{i}$ ( $i=1,2,3,4)$ are the midpoints of the edges of the element $T$.

Applying the Bramble-Hilbert lemma and

$$
\left|\frac{\partial^{2} M_{i}}{\partial x_{i} \partial x_{j}}\right| \leq C h_{k}^{-1}, \quad\left|\frac{\partial^{2} N_{i}}{\partial x_{i} \partial x_{j}}\right| \leq C h_{k}^{-1}, \quad i, j,=1,2,
$$

the following inequality holds:

$$
\left|\pi_{k} v-I_{k-1}^{k} v\right|_{2, T} \leq C\left(|v|_{2, T_{1}}+|v|_{2, T_{2}}+|v|_{2, T_{3}}\right) .
$$

Summing the above inequality for $T \in \tau_{k}$, we get

$$
\left\|\pi_{k} v-I_{k-1}^{k} v\right\|_{k} \leq C\|v\|_{k-1} \text {. }
$$

Lemma 2.1 follows from inequalities (3.1)-(3.3).

LEMMA 3.2 (Approximation Property). For any $v \in V_{k-1}+H^{2}(\Omega) \cap H_{0}^{2}(\Omega)$, there exists a constant $C$ such that

$$
\left\|v^{I}-\left(I_{k-1}^{k} v\right)^{I}\right\|_{H^{1}} \leq C h_{k}\|v\|_{k-1}
$$

and

$$
\left\|\pi_{k} w-I_{k-1}^{k}\left(\pi_{k-1} w\right)\right\|_{k} \leq C h_{k}|w|_{H^{3}}, \quad \forall w \in H^{3}(\Omega) \cap H_{0}^{2}(\Omega) .
$$

PROOF. The first inequality can be obtained as Corollary 1 in [4].

Using Lemma 2.1, the interpolation theorem in Sobolev space and

$$
\tau_{k} w=l_{k-1}^{k} w, \quad \forall w \in H^{3}(\Omega) \cap H_{0}^{2}(\Omega),
$$

the second inequality is obtained. Thus, the result of Lemma 2.2 is proved. 


\section{The multigrid algorithm}

From the spectral theorem, there exist eigenvalues $0 \leq \lambda_{1} \leq \lambda_{2} \leq \cdots \leq \lambda_{N_{k}}$ and eigenfunctions $\phi_{1}, \phi_{2}, \ldots, \phi_{N_{k}}$, such that

$$
a_{k}\left(\phi_{i}, v\right)=\lambda_{i}\left(\phi_{i}, v\right), \quad \forall v \in V_{k},
$$

and $\left(\phi_{i}, \phi_{j}\right)=\delta_{i j}$ (the Kronecker Delta). By the inverse inequality (see [7]) we have

$$
\lambda_{n_{k}} \leq C h_{k}^{4} \text {. }
$$

Assuming $v=\sum_{i=1}^{N_{k}} c_{i} \phi_{i}$, the norm $\||\cdot|\|_{s, k}$ is defined as follows:

$$
\|v\|_{s, k}^{2}:=\sum_{i=1}^{N_{k}} \lambda_{i}^{s / 2} c_{i}^{2}, \quad s \in R .
$$

Obviously, $\|v\|_{0, k}=\|v\|_{L^{2}}$ and \|\|$v\left\|_{2, k}=\right\| v \|_{k}$.

Let $z_{0}$ be an initial guess value of the solution. Then an approximate solution $M G\left(k, z_{0}, G\right)$ of the multigrid algorithm is defined by the following problem: Find $z \in V_{k}$, such that

$$
a_{k}(z, v)=G(v), \quad \forall v \in V_{k}, \quad G \in V_{k}^{\prime},
$$

where $V_{k}^{\prime}$ denotes the conjugate space of $V_{k}$.

For $k=1, M G\left(1, z_{0}, G\right)$ is the solution of (4.2) obtained from a direct method. For $k>1, M G\left(k, z_{0}, v\right):=z_{m}+I_{k-1}^{k} q_{p}$, where $z_{m}$ can be obtained by $m$ smoothing steps:

$$
\left(z_{i}-z_{i-1}, v\right)=\Lambda_{k}^{-1}\left(G(v)-a_{k}\left(z_{i-1}, v\right)\right), \quad \forall v \in V_{k},
$$

where $1 \leq i \leq m, \Lambda=C h_{k}^{-4}$, (see (4.1)). The quantity $q_{p}$ is obtained by the $(k-1)$ th-level iteration being performed $p$ times $(p=2,3)$, that is,

$$
q_{0}=0, \quad q_{i}=M G\left(k-1, q_{i-1}, \bar{G}\right), \quad 1 \leq i \leq p,
$$

where

$$
\bar{G}=G\left(I_{k-1}^{k} v\right)-a_{k}\left(z_{m}, I_{k-1}^{k} v\right)=a_{k}\left(z-z_{0}, I_{k-1}^{k} v\right), \quad \forall v \in V_{k-1} .
$$

The full multigrid method is defined as follows. Let $\hat{u}_{1}$ be the solution by a direct method. The approximations $\hat{u}_{k}(k \geq 2)$ are obtained recursively by

$$
\begin{aligned}
u_{0}^{i} & =I_{j-1}^{j} \hat{u}_{j-1}, \\
u_{l}^{j} & =M G\left(j, u_{l-1}^{j}, G\right), \quad 1 \leq l \leq r, \quad G(v)=\int_{G} f v d x_{1} d x_{2}, \\
\hat{u}_{j} & =u_{r}^{j},
\end{aligned}
$$

where $r$ is a positive integer to be determined. 


\section{The convergence analysis}

First, we give the following statement:

$\left(S_{k}\right)$ When the $k$ th-level iteration is applied to the variational problem, we have

$$
\left\|z-M G\left(k, z_{0}, G\right)\right\|_{k} \leq \gamma\left\|z-z_{0}\right\|_{k},
$$

where $\gamma \in(0,1)$ is a constant independent of $k$.

THEOREM 5.1. If the number of smoothing steps $m$ is large enough, then we have in the energy norm

$$
\left(S_{k-1}\right) \rightarrow\left(S_{k}\right)
$$

PROOF. Let $e_{0}:=z-z_{0}, e_{i}:=z-z_{i}(1 \leq i \leq m+1)$. Recall that $q$ satisfies

$$
a_{k-1}(q, v)=\bar{G}(v)=a_{k}\left(e_{0}, I_{k-1}^{k} v\right), \quad \forall v \in V_{k-1} .
$$

Let $\bar{z}_{1}:=z_{0}+l_{k-1}^{k} q, \bar{e}_{1}:=z-\bar{z}_{1}$, then $\bar{z}_{i}(2 \leq i \leq m+1)$ can be obtained recursively by

$$
\left(\bar{z}_{i}-\bar{z}_{i-1}, v\right)=\frac{1}{\Lambda_{k}}\left(G(v)-a_{k}\left(\bar{z}_{i-1}, v\right)\right), \quad \forall v \in V_{k} .
$$

Thus we can get

$$
\left\|z-M G\left(k, z_{0}, G\right)\right\|_{k}=\left\|z-z_{m+1}\right\|_{k}=\left\|e_{m+1}\right\| \leq\left\|e_{m+1}-\bar{z}_{m+1}\right\|+\left\|\bar{z}_{m+1}\right\|_{k} .
$$

From (4.3) and (5.1), we have that

$$
\left(e_{i}-e_{i-1}, v\right)=-\frac{1}{\Lambda_{k}} a_{k}\left(e_{i-1}, v\right)
$$

and

$$
\left(\bar{e}_{i}-\bar{e}_{i-1}, v\right)=-\frac{1}{\Lambda_{k}} a_{k}\left(\bar{e}_{i-1}, v\right) .
$$

Subtracting (5.4) from (5.3) implies that

$$
\left(e_{i}-\bar{e}_{i}-e_{i-1}+\bar{e}_{i-1}, v\right)=-\frac{1}{\Lambda_{k}}\left(e_{i-1}-\bar{e}_{i-1}, v\right) .
$$

Let $p_{i}=e_{i}-\bar{e}_{i}$, then

$$
\left(p_{i}-p_{i-1}, v\right)=-\frac{1}{\Lambda} a_{k}\left(p_{i-1}, v\right), \quad \forall v \in V_{k}
$$


Assume that $p_{1}=\sum_{i=1}^{n_{k}} c_{i} \phi_{i}$, then $p_{m+1}=\sum_{i=1}^{n_{k}} c_{i}\left(1-\lambda_{i} / \Lambda_{k}\right)^{m} \phi$. Thus

$$
\left\|e_{m+1}-\bar{e}_{m+1}\right\|_{k}^{2}=\left\|p_{m+1}\right\|_{k}^{2}=\sum_{i=1}^{n_{k}} c_{i}^{2}\left(1-\frac{\lambda_{i}}{\Lambda}\right)^{2 m} \lambda_{i} .
$$

Using $\lambda_{i} \leq \Lambda_{k} \leq C h_{k}^{-4}$, we finally get $\left\|e_{m+1}-\bar{z}_{m+1}\right\|_{k}^{2} \leq\left\|p_{1}\right\|_{k}^{2}=\left\|e_{1}-\bar{z}_{1}\right\|_{k}^{2}$, that is,

$$
\left\|e_{m+1}-\bar{e}_{m+1}\right\|_{k} \leq\left\|e_{1}-\bar{e}_{1}\right\|_{k} \text {. }
$$

Using $\left\|e_{1}-\bar{e}_{1}\right\|_{k}=\left\|I_{k-1}^{k}\left(q_{p}-q\right)\right\|_{k}$ and Lemma 2.1, we have the inequality

$$
\left\|e_{1}-\bar{e}_{1}\right\|_{k} \leq C \gamma^{p}\|q\|_{k-1} .
$$

On the other hand, $q$ satisfies the equation

$$
a_{k-1}(q, v)=a_{k}\left(e_{0}, I_{k-1}^{k} v\right), \quad \forall v \in V_{k-1} .
$$

Thus we have

$$
\|q\|_{k-1} \leq\left\|e_{0}\right\|_{k}
$$

Combining (5.5), (5.6) and (5.7) implies

$$
\left\|e_{m+1}-\bar{e}_{m+1}\right\|_{k} \leq C \gamma^{p}\left\|e_{0}\right\|_{k}
$$

Now we analyse the term $\left\|\bar{e}_{m+1}\right\|_{k}$ of (5.2). Let $\bar{e}_{1}=\sum_{i=1}^{n_{k}} c_{i} \phi_{i}$, then we get from the above analysis that

$$
\bar{e}_{m+1}=\sum_{i=1}^{n_{k}} c_{i}\left(1-\frac{\lambda_{i}}{\Lambda_{k}}\right)^{m} \phi_{i}
$$

In view of the definition of $\left.\||\cdot|\|\right|_{s, k}$, we obtain

$$
\left\|e_{m+1}\right\|_{k}^{2}=\sum_{i=1}^{n_{k}} c_{i}^{2}\left(1-\frac{\lambda_{i}}{\Lambda_{k}}\right)^{2 m} \lambda \leq C \frac{h_{k}^{-2}}{\sqrt{4 m+1}}\left\|\bar{e}_{1}\right\|_{1, k}^{2},
$$

that is,

$$
\left\|\bar{e}_{m+1}\right\|_{k} \leq C \frac{h_{k}^{-1}}{\sqrt[4]{4 m+1}}\left\|\bar{e}_{1}\right\| \|_{1, k}
$$

From [4] we know that

$$
\left\|\bar{e}_{1}\right\|_{1, k} \leq C\left(\left\|\bar{e}_{1}^{I}\right\|_{H^{\prime}}+h_{k}\left\|\bar{e}_{1}\right\|_{k}\right) .
$$

Let $\bar{e}_{1}^{I} \in H_{0}^{1}(\Omega)$, then $-\Delta \bar{e}_{1}^{I}=\phi \in H^{-1}(\Omega)$ and

$$
\|\phi\|_{H^{-1}}=\sup _{\varphi \in H_{0}^{\prime}} \frac{|(\phi, \varphi)|}{\|\varphi\|_{H_{0}^{\prime}(\Omega)}} \text {. }
$$


By means of Green's formula and the Cauchy-Schwarz inequality, we get

$$
|(\phi, \varphi)| \leq\left|\bar{e}_{1}^{I}\right|_{H^{\prime}}\|\varphi\|_{H^{\prime}} .
$$

Thus

$$
\|\phi\|_{H^{-1}(\Omega)} \leq\left|\bar{e}_{1}^{I}\right|_{H^{\prime}(\Omega)} .
$$

Now let $\xi \in H_{0}^{2}(\Omega) \cap H^{3}(\Omega)$ be the solution of the biharmonic equation with righthand side $\phi \in H^{-1}$, that is,

$$
\begin{cases}\Delta^{2} \xi=\phi & \text { in } \Omega, \\ \xi=\partial \xi / \partial n & \text { on } \partial \Omega .\end{cases}
$$

From the elliptic regularity, one gets

$$
\|\xi\|_{H^{3}} \leq C\|\phi\|_{H^{-1}} \leq C\left|\bar{e}_{1}^{I}\right|_{H^{\prime}} .
$$

Assume $\xi_{k} \in V_{k}$ and $\xi_{k-1} \in V_{k-1}$ satisfy

$$
a_{k}\left(\xi_{k}, v\right)=\left(\phi, v^{I}\right), \quad \forall v \in V_{k}
$$

and

$$
a_{k-1}\left(\xi_{k-1}, v\right)=\left(\phi, v^{l}\right), \quad \forall v \in V_{k-1}
$$

Then we have

$$
\left\|\xi-\xi_{k}\right\|_{k} \leq C h_{k}|\xi|_{H^{3}}, \quad\left\|\xi-\xi_{k-1}\right\|_{k-1} \leq C h_{k-1}|\xi|_{H^{3}}
$$

and

$$
\left|\bar{e}_{1}^{I}\right|_{H^{\prime}}=\left[\left(\phi, \bar{e}_{1}^{I}\right)-a_{k}\left(\xi, e_{0}-q\right)\right]+a_{k}\left(\xi, e_{0}-q\right):=I_{1}+I_{2} .
$$

Let

$$
\begin{aligned}
I_{1} & =\left(\phi, \bar{e}_{1}^{I}\right)-a_{k}\left(\xi, e_{0}-q\right) \\
& =a_{k}\left(\xi_{k}-\xi, e_{0}\right)+a_{k-1}\left(\xi-\xi_{k-1}, q\right)-\left(\phi,\left(I_{k-1}^{k} q\right)^{I}-q^{I}\right),
\end{aligned}
$$

then it holds that

$$
\left|I_{1}\right| \leq C h_{k}\left|\bar{e}_{1}^{\prime}\right|_{H^{\prime}}\left\|e_{0}\right\|_{k}
$$

Let

$$
\begin{aligned}
I_{2}=a_{k}\left(\xi, e_{0}-q\right)= & a_{k}\left(\xi-\pi_{k} \xi, e_{0}\right)+a_{k}\left(\pi_{k} \xi-I_{k-1}^{k}\left(\pi_{k-1} \xi\right), e_{0}\right) \\
& -a_{k-1}\left(\xi-\pi_{k-1} \xi, q\right)
\end{aligned}
$$

then the approximation property implies $\left|I_{2}\right| \leq C h_{k}\left|\bar{e}_{1}^{\prime}\right|_{H^{\prime}}\left\|e_{0}\right\|_{k}$. Therefore the following inequality holds:

$$
\left|\bar{e}_{1}^{I}\right|_{H^{1}} \leq C h_{k}\left\|e_{0}\right\|_{k}
$$


However,

$$
\left\|\bar{e}_{1}^{I}\right\|_{k}=\left\|z-z_{0}\right\|_{k}=\left\|e_{0}-I_{k-1}^{k} q\right\|_{k} \leq\left\|e_{0}\right\|_{k}+\left\|I_{k-1}^{k} q\right\|_{k} \leq C\left\|e_{0}\right\|_{k} .
$$

In view of (5.10), we finally get

$$
\left\|\bar{e}_{1} \mid\right\|_{1, k} \leq C h_{k}\left\|e_{0}\right\|_{k} .
$$

Combining (5.9) and (5.12), we obtain

$$
\left\|\vec{e}_{m+1}\right\|_{k} \leq \frac{C}{\sqrt[4]{4 m+1}}\left\|e_{0}\right\|_{k} .
$$

It follows from (5.8) and (5.13) that

$$
\left\|z-M G\left(k, z_{0}, G\right)\right\|_{k} \leq\left(C \gamma^{p}+\frac{C}{\sqrt[4]{4 m+1}}\right)\left\|e_{0}\right\|_{k} .
$$

Choosing $\gamma \in(0,1)$ and $m$ such that

$$
\left(C \gamma^{p}+\frac{C}{\sqrt[4]{4 m+1}}\right) \leq \gamma
$$

we have $\left\|z-M G\left(k, z_{0}, G\right)\right\|_{k} \leq \gamma\left\|e_{0}\right\|_{k}$. The proof is now complete.

THEOREM 5.2. If $\hat{u}_{k}$ and $\hat{u}_{k}^{*}$ are the solutions of the full multigrid algorithm for the problems (2.4) and (2.6), then there exists a constant $C$ such that

$$
\left\|u-\hat{u}_{k}\right\|_{k} \leq C h_{k}\left(|u|_{H^{3}}+h_{k}\|f\|_{L^{2}}\right)
$$

and

$$
\left\|u-\hat{u}_{k}^{*}\right\|_{k} \leq C h_{k}|u|_{H^{3}}
$$

PROOF. We only prove the first inequality, since the proof of the second inequality is similar to the first. By (2.5) and Theorem 5.1, one obtains

$$
\begin{aligned}
\left\|u-\hat{u}_{k}\right\|_{k} & =\left\|u-u_{r}^{k}\right\|_{k} \\
& \leq\left\|u-u_{k}\right\|_{k}+\left\|u_{k}-u_{r}^{k}\right\|_{k} \\
& \leq\left\|u-u_{k}\right\|_{k}+C \gamma^{r}\left\|u_{k}-I_{k-1}^{k} \hat{u}_{k-1}\right\|_{k} \\
& \leq C\left[h_{k}|u|_{2}+h_{k}^{2}\|f\|_{L^{2}}\right]+C \gamma^{r}\left\|u_{k}-I_{k-1}^{k} \hat{u}_{k-1}\right\|_{k} .
\end{aligned}
$$

But

$$
\begin{aligned}
\left\|u_{k}-I_{k-1}^{k} \hat{u}_{k-1}\right\|_{k} \leq & \left\|u_{k}-u\right\|_{k}+\left\|u-\pi_{k} u\right\|_{k}+\left\|\pi_{k} u-I_{k-1}^{k}\left(\pi_{k-1} u\right)\right\|_{k} \\
& +\left\|I_{k-1}^{k}\left(\pi_{k-1} u\right)-I_{k-1}^{k} \hat{u}_{k-1}\right\|_{k} \\
\leq & C\left(h_{k}|u|_{3}+h_{k}^{2}\|f\|_{L^{2}}\right)+\left\|u-\hat{u}_{k-1}\right\|_{k-1} .
\end{aligned}
$$


Thus

$$
\left\|u-\hat{u}_{k}\right\|_{k} \leq C h_{k}\left(|u|_{H^{3}}+h_{k}\|f\|_{L^{2}}\right)+\frac{C h_{k} \gamma^{r}}{1-2 C \gamma^{r}}\left(|u|_{H^{3}}+h_{k}\|f\|_{L^{2}}\right) .
$$

Choosing $\gamma$ such that $1-2 C \gamma^{r}>0$, we then have

$$
\left\|u-\hat{u}_{k}\right\|_{k} \leq C\left(h_{k}|u|_{H^{3}}+h_{k}^{2}\|f\|_{L^{2}}\right) .
$$

Theorem 5.2 follows by the above analysis.

\section{Acknowledgement}

This work was supported by The Science Foundation of China.

\section{References}

[1] H. Blum and R. Rannacher, "On the boundary value problem of the biharmonic operator on domains with angular corners", Math. Meth. Appl. Sci. 2 (1980) 556-581.

[2] D. Braess and R. Verfurth, "Multigrid methods for nonconforming finite element methods", SIAM J. Numer. Anal. 27 (1990) 979-986.

[3] J. Bramble, J. Pasciak and J. Xu, "The analysis of multigrid algorithms with nonnested spaces or non-inherited quadratic fornns", Math. Comp. 56 (1991) 1-34.

[4] S. C. Brenner, "An optimal-order nonconforming multigrid method for the biharmonic equation", SIAM J. Numer. Anal. 26 (1989) 1124-1138.

[5] Zhi-Qing Cai, C. I. Golastein and J. E. Pasciak, "Multilevel iteration for mixed finite element systems with penalty", SIAM J. Sci. Comput. 14 (1993) 1072-1088.

[6] Qian-Shun Chang and Lin-Bao Xu, "A numerical method for a system of generalized nonlinear Schrödinger equations", J. Comput. Math. 4 (1986) 191-199.

[7] P. G. Ciarlet, The finite element method for elliptic problem (North-Holland, Amsterdam, 1978).

[8] C. I. Golastein, "Multigrid methods for elliptic problems in unbounded domains", SIAM J. Numer. Anal. 30 (1993) 159-183.

[9] P. Lascaux and P. Lesaint, "Some nonconforming finite elements for the plate bending problem", RAIRO. Anal Numer. 9 (1975) 9-53.

[10] Zhong-Ci Shi, "Construction and analysis of a new energy-orthogonal unconventional plate element", J. Comput. Math. 8 (1990) 75-91.

[11] Shu-Zi Zhou and Feng Gang, "A multigrid method for the Zienkiewicz element approximation of harmonic equations", J. Hunan Unv. XueBao 20 (1993) 1-6. 